\title{
Bacteria and sputum inflammatory cell counts; a COPD cohort analysis
}

\author{
Augusta S. Beech ${ }^{1,2^{*}}$, Simon Lea', Umme Kolsum', Zhang Wang ${ }^{3}$, Bruce E. Miller ${ }^{4}$, Gavin C. Donaldson ${ }^{5}$, \\ Jadwiga A. Wedzicha ${ }^{5}$, Christopher E. Brightling ${ }^{6}$ and Dave Singh ${ }^{1,2}$
}

\begin{abstract}
Background: There is evidence that bacterial colonisation in chronic obstructive pulmonary disease (COPD) is associated with increased neutrophilic airway inflammation. This study tested the hypothesis that different bacterial phyla and species cause different inflammatory profiles in COPD patients.

Methods: Sputum was analysed by quantitative polymerase chain reaction (qPCR) to quantify bacterial load and $16 \mathrm{~S}$ rRNA gene sequencing to identify taxonomic composition. Sputum differential cell counts (DCC) and blood DCC were obtained at baseline and 6 months. Patients were categorised into five groups based on bacterial load defined by genome copies/ml of $\geq 1 \times 10^{4}$, no colonisation and colonisation by Haemophilus influenzae (H. influenzae), Moraxella catarrhalis (M. catarrhalis), Streptococcus pneumoniae (S. pneumoniae), or $>1$ potentially pathogenic microorganism (PPM).

Results: We observed an increase in sputum neutrophil (\%), blood neutrophil (\%) and neutrophil-lymphocyte ratio (NLR) in patients colonised with $H$. influenzae $(82.6,67.1$, and 3.29 respectively) compared to those without PPM colonisation at baseline (69.5, 63.51 and 2.56 respectively) ( $p<0.05$ for all analyses), with similar findings at 6 months. The bacterial load of $H$. influenzae and Haemophilus determined by qPCR and 16s rRNA gene sequencing respectively, and sputum neutrophil \% were positively correlated between baseline and 6 months visits ( $p<0.0001,0.0150$ and 0.0002 with $r=0.53,0.33$ and 0.44 respectively).
\end{abstract}

Conclusions: These results demonstrate a subgroup of COPD patients with persistent $\mathrm{H}$. influenzae colonisation that is associated with increased airway and systemic neutrophilic airway inflammation, and less eosinophilic airway inflammation.

Keywords: Microbiome, COPD, Haemophilus influenzae, Sputum, Eosinophil

\section{Background}

Chronic obstructive pulmonary disease (COPD) is a heterogeneous disease characterised by airflow obstruction and persistent airway inflammation [1]. COPD patients show increased susceptibility to bacterial infection, through mechanisms such as decreased bacterial

\footnotetext{
*Correspondence: gbeech@meu.org.uk

${ }^{2}$ Medicines Evaluation Unit, University of Manchester, Manchester

University NHS Foundation Trust, Southmoor Road, Manchester M23 9QZ, UK

Full list of author information is available at the end of the article
}

phagocytosis [2]. Chronic bacterial airway colonisation may occur with potentially pathogenic microorganisms (PPMs) including Haemophilus influenzae (H. influenzae), Moraxella catarrhalis (M. catarrhalis) and Streptococcus pneumoniae (S. pneumoniae) [3-5].

The presence of bacterial infection in COPD patients during the stable state (i.e. not during exacerbations) is associated with increased airway neutrophil numbers $[6$, 7]. Furthermore, it appears that different bacterial phyla are associated with different profiles of airway inflammation in COPD patients; raised blood and sputum eosinophils are associated with increased presence of the original author(s) and the source, provide a link to the Creative Commons licence, and indicate if changes were made. The images or other third party material in this article are included in the article's Creative Commons licence, unless indicated otherwise in a credit line to the material. If material is not included in the article's Creative Commons licence and your intended use is not permitted by statutory regulation or exceeds the permitted use, you will need to obtain permission directly from the copyright holder. To view a copy of this licence, visit http://creativecommons.org/licenses/by/4.0/. The Creative Commons Public Domain Dedication waiver (http://creativeco mmons.org/publicdomain/zero/1.0/) applies to the data made available in this article, unless otherwise stated in a credit line to the data. 
Bacteroidetes phylum [8], while low sputum eosinophil levels have been associated with increased $H$. influenzae presence [9]. Additionally, a study of microbiome, transcriptome and proteome profiling showed that $H$. influenzae presence in the stable state was associated with a unique profile of inflammation, including increased sputum neutrophil counts $[10,11]$.

The analyses of randomised clinical trials have demonstrated that greater inhaled corticosteroid (ICS) benefits are observed at higher blood eosinophil counts [12]. Consequently, the Global initiative for the management of chronic Obstructive Lung Disease (GOLD) report recommends the use of blood eosinophil counts to guide ICS treatment in COPD patients with a history of exacerbations [1]. Higher blood eosinophil counts in COPD patients are associated with increased eosinophil numbers in bronchial tissue, broncho-alveolar lavage and sputum, increased levels of T2 cytokines and greater basement membrane thickening [13]. The mechanistic reasons for increased ICS effects at higher blood eosinophil counts may be related to an inflammatory profile associated with increased levels of T2 cytokines, but an association with a different microbiome profile may also be important [10].

Using the COPDMAP cohort, we have further studied the relationship between sputum cell counts and bacterial species in the stable state. We evaluated the stability of the relationship between the microbiome and airway inflammation using repeated samples at 6 months, primarily focusing on bacterial load measured by quantitative polymerase chain reaction (qPCR) and sputum cell counts. Associations between these parameters and both 16S rRNA-gene based microbiome analysis and blood leucocyte counts are also reported. A focus of the analysis of repeated samples was to investigate the persistence of bacterial colonisation over 6 months, and its associations with sputum cell counts and clinical characteristics.

\section{Methods \\ Subjects}

COPD patients aged $\geq 40$ years were recruited at 3 sites (Manchester, Leicester and London) into the COPDMAP prospective observational cohort study. Patients had a physician diagnosis of COPD, post-bronchodilator forced expiratory volume in $1 \mathrm{~s}\left(\mathrm{FEV}_{1}\right) /$ forced vital capacity (FVC) ratio $<0.7, \geq 10$ pack year smoking history and no previous asthma diagnosis. The patients included in this analysis were those who provided a sputum sample for bacterial quantification at $\geq 1$ visit over 12 months. All patients provided written informed consent using protocols approved by the local Ethics Committees (11/ L0/1630; 10/H/1003/108; 07/H0406/157).

\section{Study design}

Patient visits were during stable state at baseline and at 6 months (stable defined as no symptom-defined exacerbation in the 4 weeks preceding sampling). Patients who received maintenance oral corticosteroid or antibiotic therapy were excluded from analysis. Symptoms were assessed using the COPD assessment test (CAT) [14]. Health related quality of life was assessed using the St George's respiratory Questionnaire (SGRQ-C) [15]. Lung function measurements were performed according to guidelines [16]. Spontaneous or induced sputum was obtained as previously described [17]. Blood samples were sent to local hospital laboratories for analysis of differential cell counts (DCC). A total of 236 patients were included in this analysis, the number of patients included in different sub-analyses was dependent on the availability of specific data relevant to that sub-analyses; 236 patients produced a sputum sample for bacterial analysis using qPCR at baseline; 226 had qPCR bacterial quantification and blood DCC; 145 had qPCR and sputum DCC. At 6 months, there were 100 patients with qPCR bacterial quantification and blood DCC, and 69 with qPCR and sputum DCC (Additional file 1, Fig. S1).

\section{Bacterial $\mathrm{qPCR}$ and $16 \mathrm{~S}$ rRNA measurements}

Sputum induction was attempted if an insufficient spontaneous sample was produced for the analysis, approximately $18 \%$ of cases in the current analysis. Spontaneous or induced sputum was processed for qPCR detection of absolute abundance for the following bacterial species; $H$. influenzae, M. catarrhalis and S. pneumoniae as previously described [4]. Where possible, sputum was also processed for differential cell counts [18]. Patients were termed "persistently colonised" if qPCR results indicated two consecutive positive measurements over 6 months. Although qPCR detection of bacterial species was the primary analysis, sputum was also processed for $16 \mathrm{~S}$ RNA-gene based microbiome analysis of bacterial taxonomy (see online supplement).

\section{Statistical analysis}

Patients with bacterial load $\geq 1 \times 10^{4}$ copies/ml defined by qPCR were categorised as positive for PPM colonisation [6], then split into five groups; No colonisation, $H$. influenzae, S. pneumoniae, M. catarrhalis, and two or more PPMs ( $>1$ PPMs). Similar analysis was performed using the threshold $\geq 1 \times 10^{6}$ copies $/ \mathrm{ml}$. Statistical analysis for non-parametric data was performed using; Kruskal-Wallis test followed by post-test analysis with either Wilcoxon signed rank test or the Mann-Whitney U test (with correction for multiple analysis). Spearman's correlation assessed associations between variables. 
$p<0.05$ was considered statistically significant. Analyses were performed using GraphPad Prism version 7.00 (San Diego, USA). For 16S rRNA analysis, alpha diversity was assessed using Shannon index. Kruskal-Wallis test identified taxa with significantly different abundance across groups. Wilcoxon rank sum tests compared enrichment of other taxa present in $H$. influenae and S. pneumoniae groups. For beta diversity, composition dissimilarity was tested using the Bray-Curtis dissimilarity index. The false discovery rate (FDR) method adjusted $P$ values for multiple testing and significantly differentially represented bacterial taxa were identified using edgeR [10].

\section{Results}

\section{Study subjects}

The baseline demography of the cohort $(n=236)$ is shown in Table 1; the mean post-bronchodilator FEV $_{1}$ was $57.4 \%$ predicted, with no exacerbations in the previous year in $32.6 \%$ of patients, 1 exacerbation in $22.9 \%$ and $\geq 2$ exacerbations in $44.5 \%$. Mean CAT and total SGRQ scores were 18 and 47 respectively.

\section{Baseline results}

The cohort $(\mathrm{n}=236)$ was divided into five groups using qPCR detection using a threshold $\geq 1 \times 10^{4}$ genome copies $/ \mathrm{ml}$; No colonisation $(n=108), H$. influenzae only $(n=41)$, S. pneumoniae only $(n=47)$, M. catarrhalis only $(n=4)$ and $>1$ PPM $(n=36)$, referred to as NC, $H$. influenzae, S. pneumoniae, M. catarrhalis and $>1$ PPM groups respectively. The $M$. catarrhalis group were excluded from baseline analysis due to a small sample size. The $>1$ PPM group consisted of patients with colonisation of two or three PPMs; 92\% were colonised with $H$. influenzae, with $58 \%$ colonised with $H$. influenzae + S. pneumoniae, while $42 \%$ showed evidence of $M$. catarrhalis colonisation and $8.3 \%$ were colonised with all three bacterial species (for details see Additional file 1, Table S1). The total number of patients in the entire cohort with M. catarrhalis colonisation was 19 (8.1\%). There was no difference in bacterial colonisation in frequent exacerbators ( $\geq 2$ exacerbations in the previous year) compared to non-frequent exacerbators (Additional file 1, Table S2), or in patients using ICS compared to those not using ICS (data not shown).

\section{Sputum cell counts}

145 patients with qPCR data had sputum DCCs available; induced samples were more cellular based on total cell count $\left(\times 10^{6} / \mathrm{g}\right)$, with total neutrophil and macrophage counts $\left(\times 10^{6} / \mathrm{g}\right)$ also higher in induced samples in the subset with this information available $(n=112)$. However, in the total sample $(n=143)$ with available DCCs, the sputum cell percentages between induced

\begin{tabular}{|c|c|}
\hline Characteristic & $n=236$ \\
\hline Gender (\% male) & 74 \\
\hline Age & $69.5(8.3)$ \\
\hline Smoking status (current \%) & 34 \\
\hline Pack years & 47.5 [10-220] \\
\hline BMI $\left(\mathrm{kg} / \mathrm{m}^{2}\right)$ & $26.3[17.3-47.0]$ \\
\hline Exacerbations (1 year period) & $1[0-15]$ \\
\hline Post FEV ${ }_{1}(\mathrm{~L})$ & $1.5(0.6)$ \\
\hline Post FEV ${ }_{1}(\%)$ & $57.4(18.5)$ \\
\hline CAT & $18(7.5)$ \\
\hline SGRQ-C (total) & $47.0(18.2)$ \\
\hline GOLD & $2[1-4]$ \\
\hline 1 (\%) & 12.0 \\
\hline $2(\%)$ & 52.1 \\
\hline $3(\%)$ & 29.1 \\
\hline $4(\%)$ & 6.8 \\
\hline LABA only (\%) & 2.5 \\
\hline LAMA only (\%) & 5.5 \\
\hline ICS only (\%) & 1.7 \\
\hline ICS + LABA (\%) & 13.1 \\
\hline ICS + LAMA (\%) & 1.3 \\
\hline LABA + LAMA (\%) & 2.1 \\
\hline Triple (\%) & 68.2 \\
\hline No inhaled medication (\%) & 5.5 \\
\hline
\end{tabular}

Summaries are presented as percentages, mean (SD), median [range] as appropriate $\left(\mathrm{n}=236^{\mathrm{a}}\right)$

${ }^{a}$ At baseline the following data were not available for; $n=8$ Saint Georges respiratory Questionnaire (SGRQ), $n=3$ Chronic obstructive pulmonary disease assessment test (CAT), $\mathrm{n}=2$ forced expiratory volume in one second ( $\mathrm{FEV}_{1}$ )

and spontaneous samples were similar (Additional file 1, Table S3). There were no differences observed between qPCR and DCC results from induced and spontaneous samples (Additional file 1, Table S3). Sputum neutrophil \% was higher in the $H$. influenzae versus NC group; medians 82.6 and $69.5 \%$ respectively $(p=0.0004$, Fig. $1 a)$. Neutrophil percentage was also higher in the $>1$ PPM group $(79.8 \%)$, but this difference was not statistically significant $(p=0.06)$. A similar pattern showing higher sputum neutrophil absolute cell counts in the H. influen$z a e$ versus NC group was evident; medians $8.59 \times 10^{6} / \mathrm{g}$ versus $1.71 \times 10^{6} / \mathrm{g}$ respectively $(\mathrm{p}=0.0028$, Additional file 1, Table S4).

The $H$. influenzae and >1PPM groups had lower sputum eosinophil levels (medians $0.50 \%$ and $0.25 \%$ respectively) compared to the $\mathrm{NC}$ group (median $1.00 \%$; $\mathrm{p}=0.03$ and 0.04 respectively, Fig. 1b). Using $>3 \%$ to define sputum eosinophilia, the $H$. influenzae and $>1$ PPM groups had fewer patients above this threshold $(7.7 \%$ and $11.5 \%$ respectively) compared to $\mathrm{NC}(24.2 \%)$, although 
a

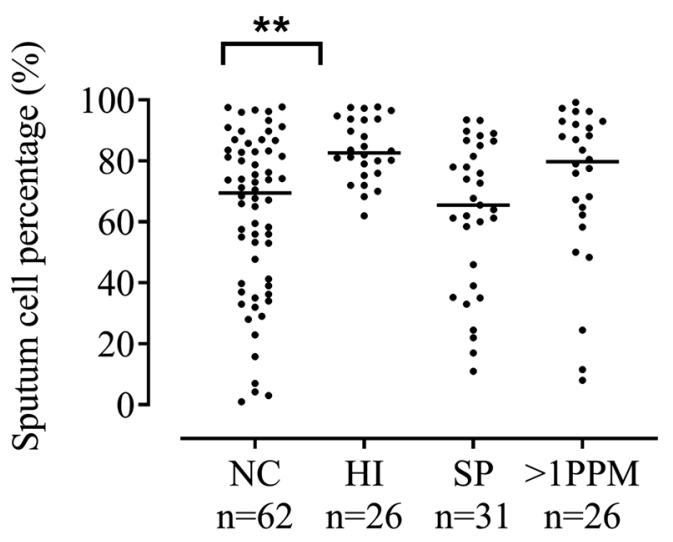

c

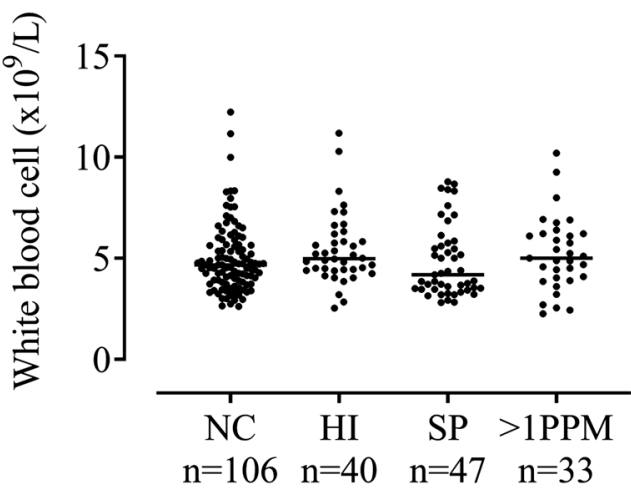

$\mathbf{e}$

Neutrophil

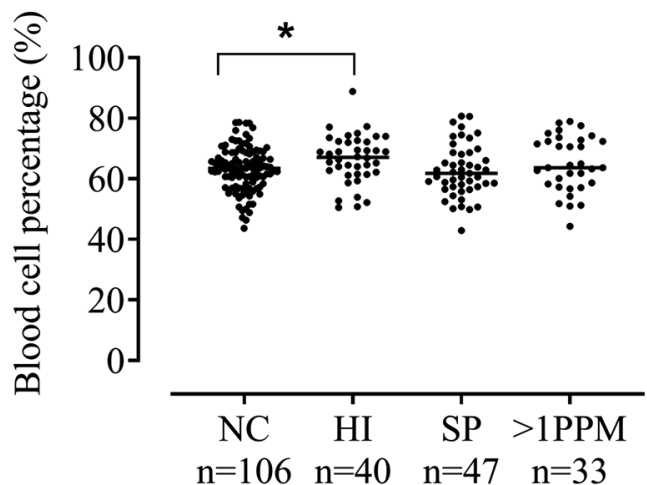

b

Eosinophil

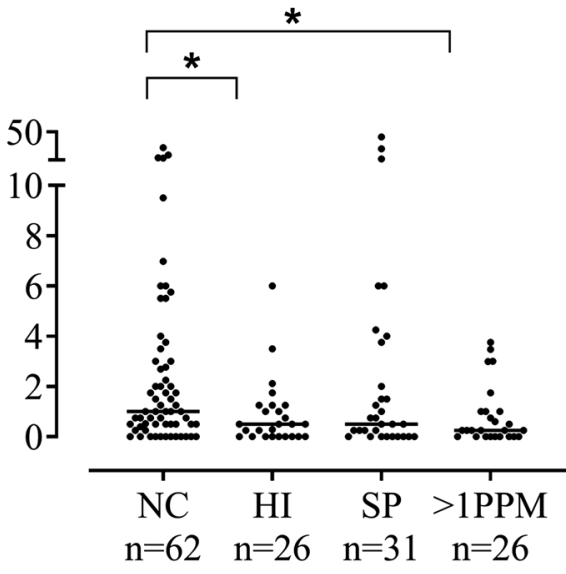

d

Eosinophil

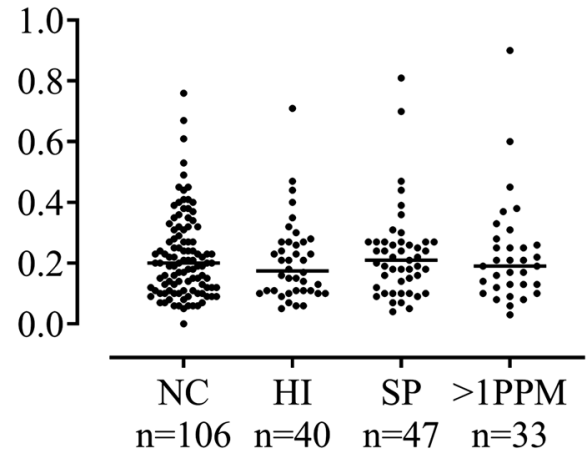

f Neutrophil-lymphocyte ratio

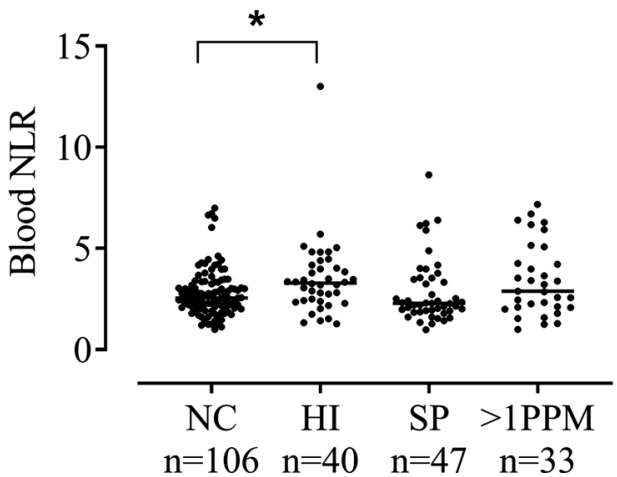

Fig. 1 The comparison of sputum and blood eosinophil and neutrophil counts between patients colonised with different PPMs. Patients were categorised into four groups based on bacterial load defined by genome copies/ml of $\geq 1 \times 10^{4}$; no colonisation (NC), colonised with Haemophilus influenzae (H), Streptococcus pneumoniae (SP) or $>1$ potentially pathogenic microorganism (PPM). Sputum neutrophil \% $(n=145)(\mathbf{a})$, sputum eosinophil \% ( $n=145)(\mathbf{b})$, blood neutrophil counts $(n=226)(\mathbf{c})$, blood eosinophil counts $(n=226)(\mathbf{d})$, blood neutrophil percentages $(n=226)(\mathbf{e})$ and blood neutrophil-lymphocyte ratio $(n=226)(\mathbf{f})$ are shown for each group. Statistical analysis was performed using Kruskal-Wallis and MannWhitney $\mathrm{U}$ adjusted for multiple comparisons. Data represent individual patients with median. ${ }^{*}{ }^{* *}$ Significant difference to no colonisation group $(p<0.05,<0.01$ respectively) 
this difference was not significant $(\mathrm{p}=0.17$; Additional file 1, Table S4). No significant differences in absolute sputum eosinophil counts were observed between groups (Additional file 1, Table S4). The clinical characteristics of the groups showed some numerical differences; $\mathrm{FEV}_{1}$ predicted was lower in the H. influenzae and S. pneumoniae groups, while SGRQ was also higher in these groups (statistical analysis shown in Additional file 1, Table S5). There were no differences in ICS use between groups.

\section{Blood cell counts}

In 226 patients with blood samples, there were no significant differences between groups for absolute blood neutrophil or eosinophil cell counts (Fig. 1c, d, Additional file 1, Table S6) or eosinophil \% (not shown). Blood neutrophil \% and neutrophil-lymphocyte ratio were significantly higher in the $H$. influenzae versus NC group (medians: neutrophils $67.1 \%$ and $63.51 \%$ respectively, $\mathrm{p}=0.0268$; neutrophil-lymphocyte ratio: 3.32 and 2.56 respectively, $\mathrm{p}=0.0086$, Fig. 1e, f). There were no significant differences in blood lymphocyte counts between groups (Additional file 1, Fig. S2).

\section{$16 \mathrm{~S}$ rRNA microbiome analysis}

Microbiome profiles for 153 patients defined using baseline qPCR results are presented in Fig. 2. An increase in relative abundance of Haemophilus $(\mathrm{FDR} \mathrm{p}=0.0004)$ and decrease in Prevotella (FDR p $=0.003$ ) and Streptococcus (FDR $\mathrm{p}=0.007$ ) was observed in both $H$. influenzae and $>1$ PPM groups compared the NC group (Fig. 2a). In contrast, the $S$. pneumoniae group was enriched with numerous other bacterial phyla including other Fusobacteria (Fusobacterium), Bacteroidetes (Prevotella), Proteobacteria (Campylobacter and Neisseria) and Actinobacteria (Rothia and Actinomyces) (FDR p <0.05). There were significant differences in Shannon indexes between groups; alpha diversity decreased in both $H$. influenzae and $>1$ PPM groups compared to NC. When analysed in relation to sputum $(\mathrm{n}=128)$ and blood cell counts $(\mathrm{n}=153)$, Shannon diversity was negatively associated with sputum neutrophil $\%(\mathrm{p}<0.01$, rho $=-0.24)$ and absolute counts $(\mathrm{p}=0.05$, rho $=-0.21)$ but not blood neutrophils (data not shown).

\section{6 month results}

\section{Sputum cell counts}

66 patients with qPCR data had sputum cell counts available. The $H$. influenzae group had significantly higher neutrophil percentages compared to the $\mathrm{NC}$ group (medians $86.6 \%$ and $69.5 \%$ respectively, $p=0.02$, Fig. 3a), with a similar pattern for absolute neutrophil counts $\left(12.89\right.$ and $1.45 \times 10^{6} / \mathrm{g}$ respectively, $\mathrm{p}=0.0048$, Additional file 1 , Table S7). The $H$. influenzae group had lower sputum eosinophil percentages compared to the $\mathrm{NC}$ group $(0.38 \%$ and $2.13 \%$ respectively, $p=0.011$, Fig. $3 b)$, with no significant differences for absolute counts.

\section{Blood cell counts}

In 100 patients with blood samples, there were no consistent differences between groups for neutrophils, eosinophils or neutrophil-lymphocyte ratio (Fig. 3c-f, Additional file 1, Table S8).

\section{Analysis using alternative qPCR threshold}

An analysis using a threshold of $\geq 1 \times 10^{6}$ copies $/ \mathrm{ml}$ to define colonisation showed a similar pattern of results to $\geq 1 \times 10^{4}$ copies $/ \mathrm{ml}$, at both baseline and 6 months; details are in the supplement (Additional file 1, Figs. S3, S4 and Tables S9, S10, S11, S12 and S13).

\section{Stability over 6 months}

The bacterial load of $H$. influenzae determined by qPCR at baseline and 6 months was positively correlated $(\mathrm{n}=122$; rho $=0.51, p<0.0001$, Fig. $4 \mathrm{a})$. Associations at baseline and 6 months were also observed using Haemophilus abundance determined by $16 \mathrm{~S}$ rRNA gene sequencing $(\mathrm{n}=54 ;$ rho $=0.33, \mathrm{p}=0.015$, Additional file 1, Fig. S6) and for sputum neutrophil $\%(n=69$; $r=0.44, p=0.0002$, Fig. $4 b$ ).

The changes in colonisation from baseline to 6 months $(n=122)$ are shown in Fig. 5 . The most common findings at baseline were $H$. influenzae $(n=21,17.2 \%)$, NC $(n=56,45.9 \%)$ or $>1 \mathrm{PPM}(n=21,17.2 \%)$. For these groups, approximately $40-50 \%$ of patients remained in the same category at 6 months. Less patients in the $S$. pneumoniae and M. catarrhalis groups remained in the same category.

Analysing patients with qPCR and sputum counts at both baseline and 6 months $(n=69)$, there were 9 with H. influenzae colonisation (Fig. 6a) and 30 with no colonisation at both visits (not shown). Patients colonised at both visits (termed "persistent colonisation") had sputum neutrophil \% that mostly remained above $60 \%$ at both visits, while non-colonised patients had a greater spread of neutrophil counts (Fig. 6b, e respectively). Patients with persistent $H$. influenzae presence had a significantly lower $\mathrm{FEV}_{1}$ compared to those without $H$. influenzae colonisation (46\% versus $61.2 \%$ predicted respectively, $\mathrm{p}=0.035$; Additional file 1, Table S14 shows clinical characteristics including exacerbations rates which were not significantly different). Five patients were colonised with H. influenzae at 1 visit only (Fig. 6c); patients who developed new $H$. influenzae colonisation showed an increase in sputum neutrophil \%, while a loss of $H$. influenzae colonisation was associated with a reduction in sputum neutrophil \% (Fig. 6d). Patients persistently colonised with 

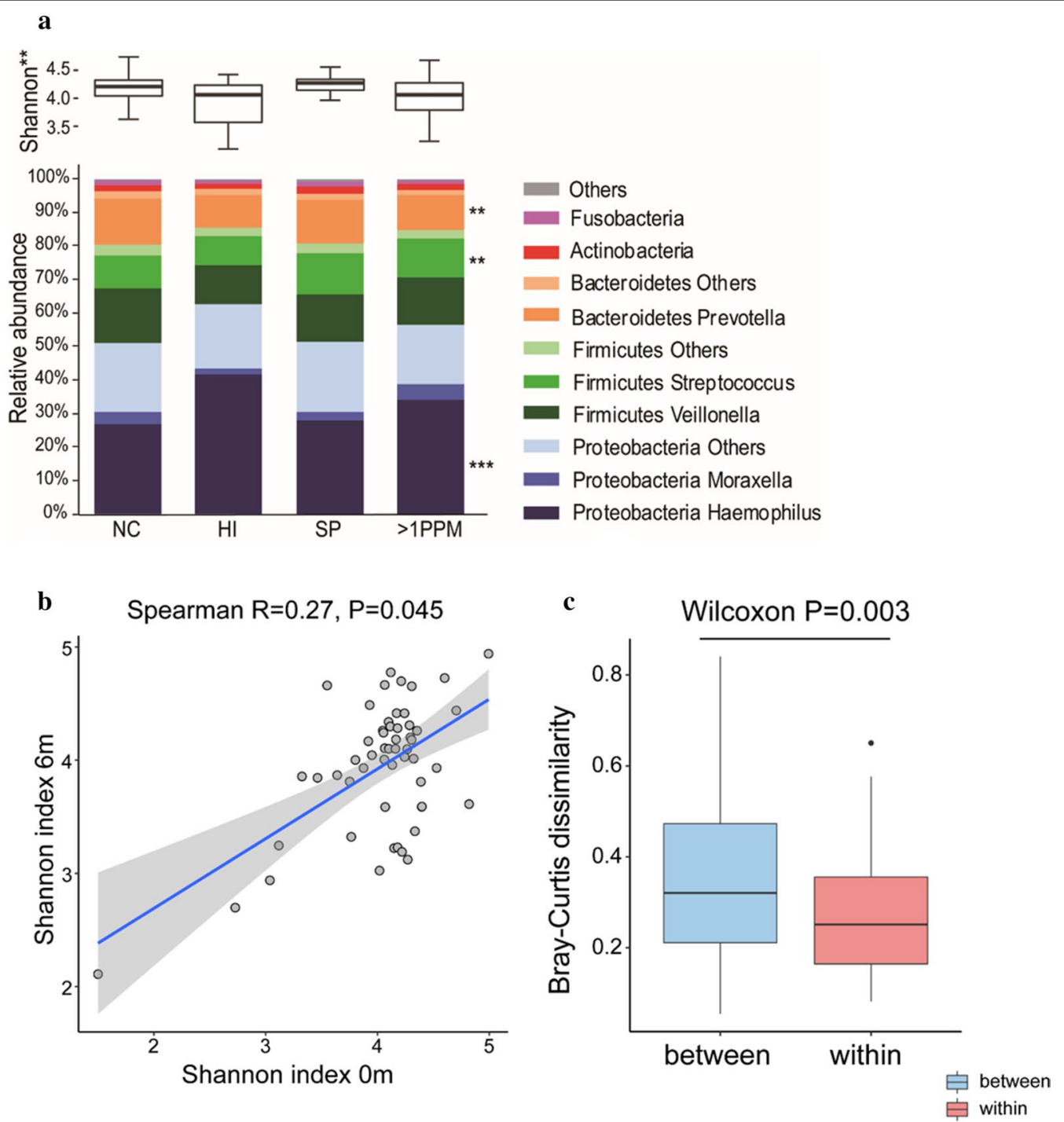

Fig. 2 Sputum microbiome profiles in COPD patients at baseline. A proportion of patients provided sufficient sample for 16S rRNA sequence analysis. Shannon Diversity and relative abundance of major bacterial taxa and genera for PPM groups defined by genome copies per $\mathrm{mL}$ of $\geq 1 \times 10^{4}$; no colonisation (NC), Haemophilus influenzae (H), Streptococcus pneumonia (SP) and $>1$ PPM $n=153$ (a), Correlation analysis for shannon diversity of paired samples at baseline and 6 months $(n=54)(\mathbf{b})$, Composition dissimilarity analysis between paired samples at baseline and 6 months, and samples from different individuals $(n=54)(\mathbf{c}) .{ }^{*}$, ** ${ }^{* * *}$ Significant difference to no colonisation group $(F D R p<0.05,<0.01$ and $<0.001$ respectively)

H. influenzae had higher sputum neutrophil and lower sputum eosinophil $(\%)(\mathrm{p}=0.011$ and 0.016 respectively; Additional file 1, Table S15). A further 16 patients were persistently colonised with $H$. influenzae $+\geq 1$ other PPM at both baseline and 6 months visits. We combined these 16 patients with the 9 patients colonised with $H$. influenzae only, and compared this group $(\mathrm{n}=25)$ to noncolonised patients. Again, there was a significantly lower $\mathrm{FEV}_{1} \%$ predicted in $H$. influenzae colonised patients compared to those without colonisation $(\mathrm{p}=0.01)$, but no difference in exacerbation rates (Additional file 1,
Table S16). Sputum neutrophil \% was significantly higher in $H$. influenzae persistently colonised patients and sputum eosinophil \% was lower $(\mathrm{p}<0.01$ and 0.03 respectively. Additional file 1, Table S17). Using eosinophil \% thresholds, a significantly lower proportion of eosinophilic patients were observed in the $H$. influenzae persistently colonised group ( $\mathrm{p}=0.01$ and $<0.01$ using 2 and $3 \%$ sputum eosinophil thresholds respectively).

The 6 month data for 16S rRNA analysis showed a similar pattern to the baseline data but with a smaller sample size $(\mathrm{n}=73)$ (Additional file 1, Figs. S5 and S6). 
a

Neutrophil

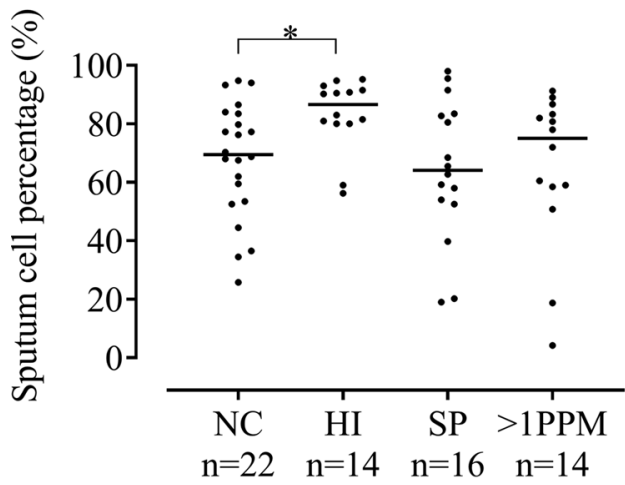

c

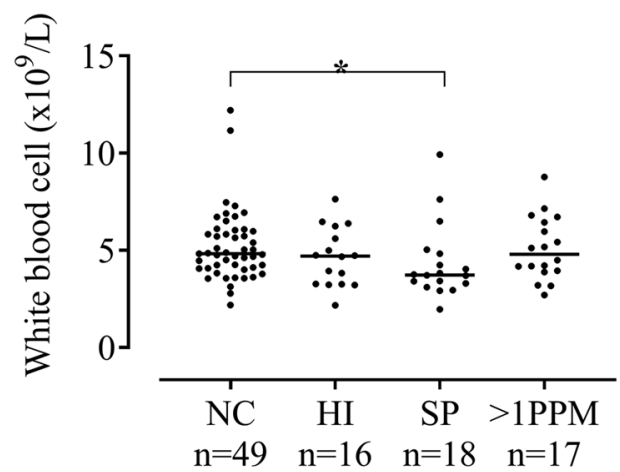

$\mathbf{e}$

Neutrophil

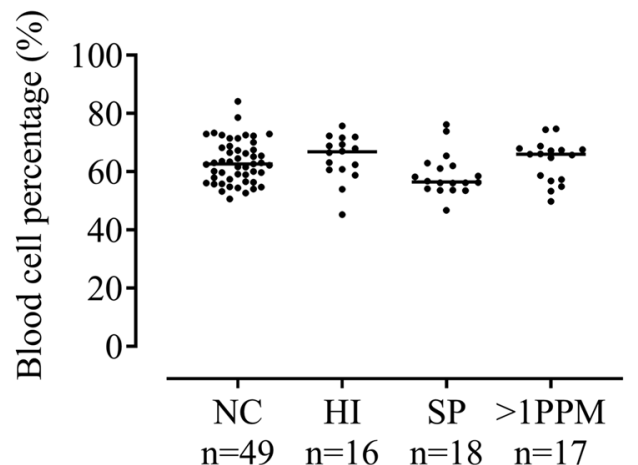

b

Eosinophil

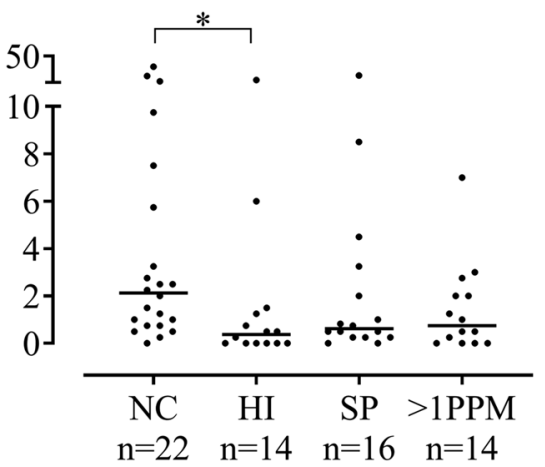

d

Eosinophil

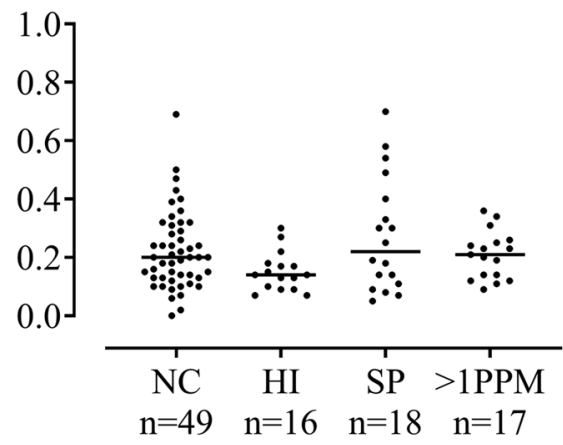

f

Neutrophil-lymphocyte ratio

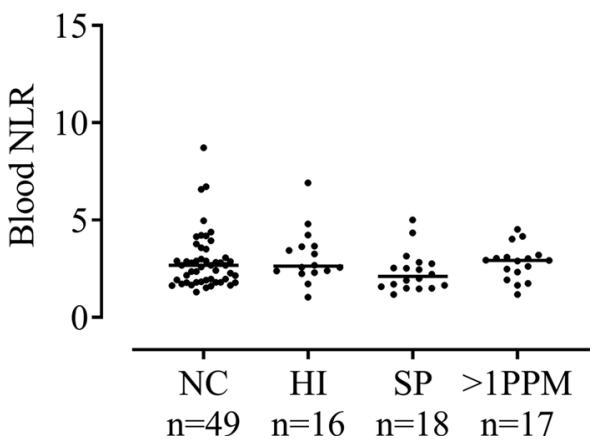

Fig. 3 The comparison of sputum and blood eosinophil and neutrophil counts between patients colonised with different PPMs at 6 months post-baseline. Patients were categorised into four groups based on bacterial load defined by genome copies/ml of $\geq 1 \times 10^{4}$; no colonisation (NC), colonised with Haemophilus influenzae (H), Streptococcus pneumoniae (SP) or $>1$ potentially pathogenic microorganism (PPM). Sputum neutrophil $\%(n=66)(\mathbf{a})$, Sputum eosinophil \% $(n=66)(\mathbf{b})$. Three patients were excluded due to colonisation with Moraxella catarrhalis at 6 months. Blood neutrophil count $(n=100)(\mathbf{c})$, Blood eosinophil count $(n=100)$ (d) Blood neutrophil \% $(n=100)(\mathbf{e})$ and Blood neutrophil-lymphocyte ratio $(n=100)(\mathbf{f})$. Statistical analysis was performed using Kruskal-Wallis and Mann-Whitney U adjusted for multiple comparisons. Data represent individual patients with median. *Significant difference to no colonisation group $(p<0.05$ respectively) 

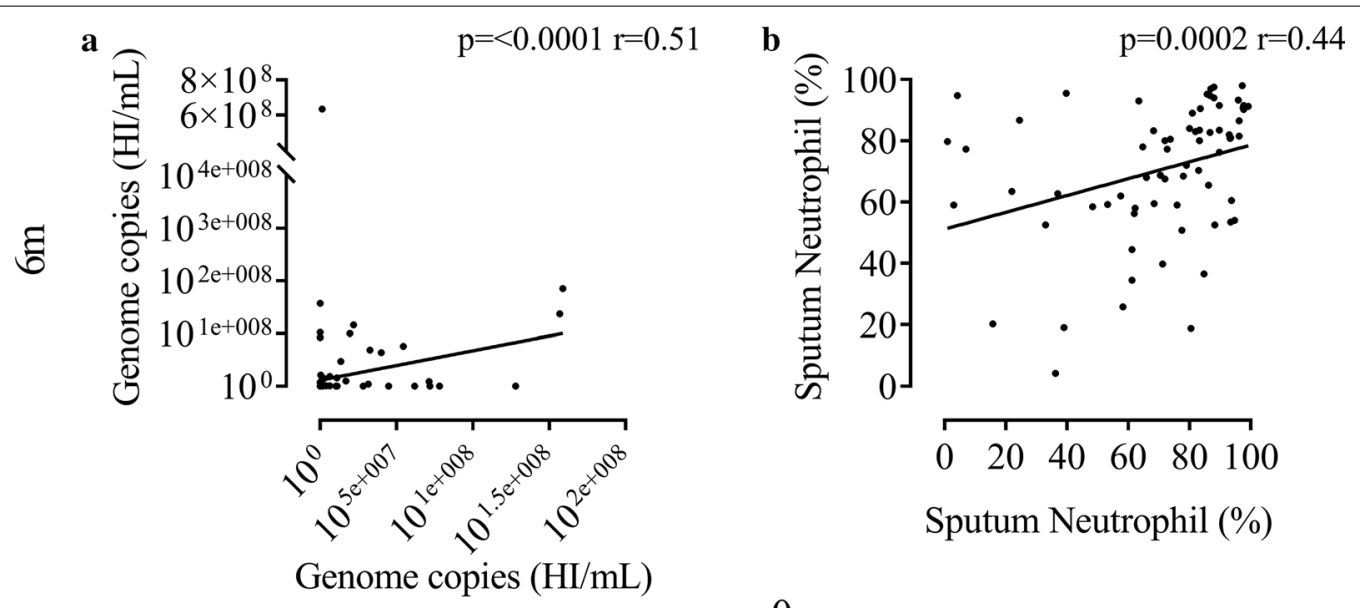

$0 \mathrm{~m}$

Fig. 4 Stability of Haemophilus influenzae presence and airway neutrophil \% over 6 months. Scatter correlation of Haemophilus influenzae (HI) genome copies/mL at baseline versus 6 months thereafter $(n=122)(\mathbf{a})$. Scatter correlation of sputum neutrophil percentage at baseline versus 6 months thereafter $(n=69)(\mathbf{b})$. Statistical analysis was performed using Spearmans Rank order correlation. Data represent individual patients with linear regression

The Shannon diversity for paired samples at baseline and $6 \mathrm{~m}$ showed a positive correlation $(\mathrm{n}=54)$ (Spearman $\mathrm{R}=0.27, \mathrm{p}=0.045$, Fig. $2 \mathrm{~b}$ ). We tested the composition dissimilarity between samples from the same individual at baseline and 6 months, and samples from different individuals. The samples from the same patients had significantly smaller Bray-Curtis dissimilarity indices than those from different individuals $(n=54)$ (Wilcoxon $\mathrm{p}=0.003$, Fig. $2 \mathrm{c}$ ), suggesting relative temporal stability of the airway microbiome in COPD.

\section{Discussion}

This analysis of the COPDMAP cohort demonstrated a subgroup of COPD patients with $H$. influenzae colonisation in the stable state, associated with increased neutrophil and decreased eosinophil numbers in sputum. Although colonisation status and microbiome changed over time in some patients, we observed that approximately $40 \%$ of patients within the $H$. influenzae group at baseline had persistent $H$. influenzae colonisation at 6 months, with a similar profile of airway inflammation. $H$. influenzae colonisation was also associated with increased blood neutrophils numbers. In contrast, there was no association between neutrophilic inflammation and $S$. pneumoniae colonisation. These results reveal a distinct COPD subgroup with $H$. influenzae colonisation that persists over time and is associated with increased neutrophilic inflammation in both the lungs and systemic circulation.

The 16S rRNA microbiome results supported the qPCR analysis, showing increased Haemophilus abundance in the $H$. influenzae group defined by qPCR at baseline, with no other genus enriched in this group. In contrast, a variety of other bacterial genus (not Haemophilus) were enriched in the S. pneumoniae group. Additionally, most of the >1PPM group (92\%) were colonised with $H$. influenzae, and there was a trend towards increased sputum neutrophil counts in this group. Overall, these qPCR and $16 \mathrm{~S}$ rRNA results indicate that Haemophilus colonisation may occur with or without the presence of other colonising bacteria, but that the presence of $H$. influenzae is the key component causing the association with neutrophil counts.

$H$. influenzae and $>1$ PPM groups displayed the greatest stability over 6 months compared to S. pneumoniae and $M$. catarrhalis. It was not possible to determine if the changes in inflammatory cell counts were solely attributed to the presence of $H$. influenzae or rather the persistence of airway colonisation in these patients, although there is evidence to support the former [10]. Significant associations were demonstrated using both qPCR and $16 \mathrm{~s}$ rRNA gene sequencing for Haemophilus measurements at baseline and 6 months. Sputum neutrophils showed a similar pattern. Changes in airway neutrophils on an individual basis over time showed remarkable concordance with that of $H$. influenzae colonisation in the subgroup $(n=69)$ with sputum cell counts and microbiology data at both baseline and 6 months. The presence of $H$. influenzae appeared to influence the sputum neutrophil percentage, with persistently higher neutrophils being observed in those with $H$. influenzae present at both visits, and concordant changes in neutrophils observed when $H$. influenzae presence changed (Fig. 6). We also observed that persistent $H$. influenzae colonisation was 


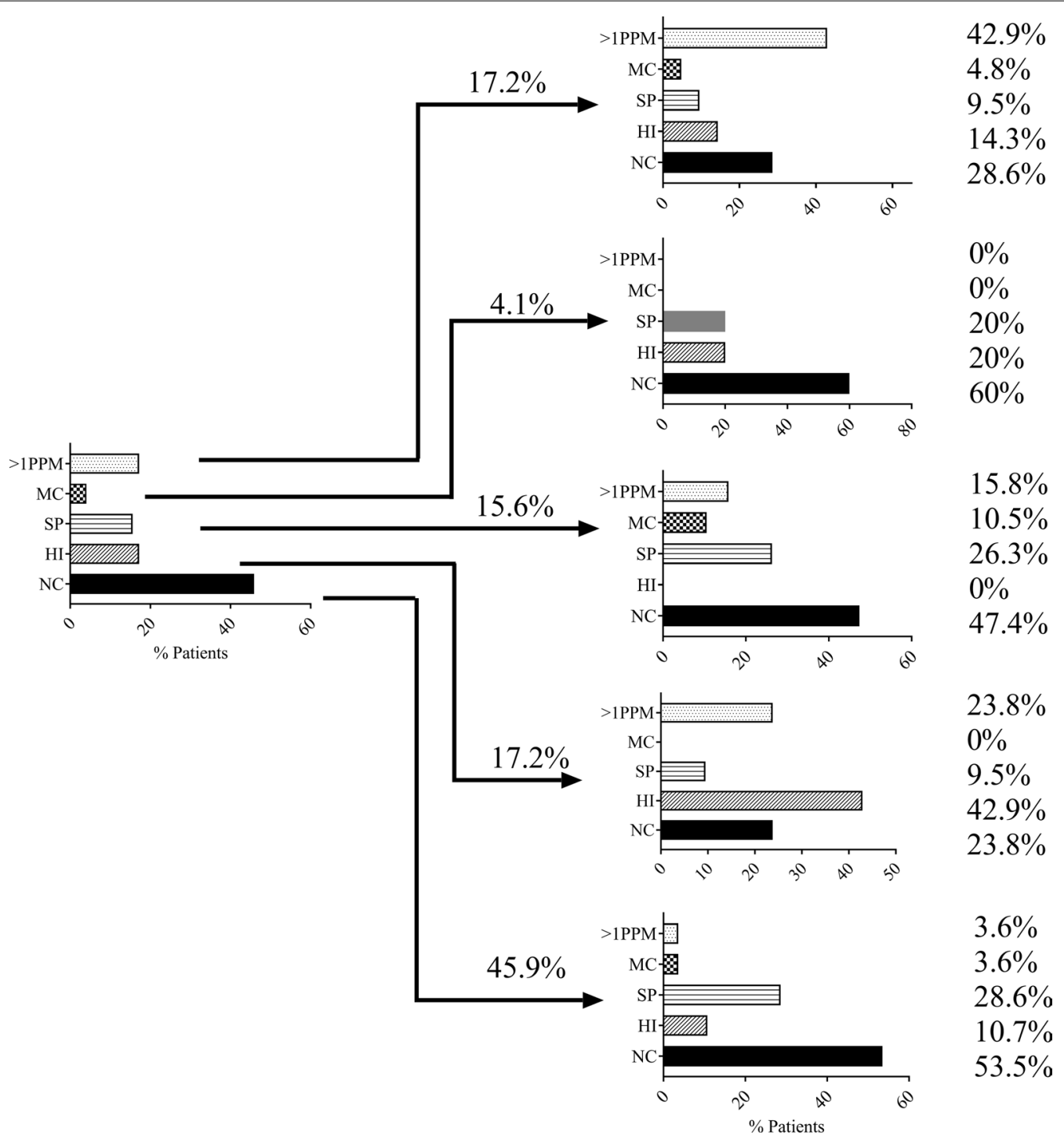

Fig. 5 Changes in airway colonisation of respective potentially pathogenic microorganisms over 6 months. Patients were categorised into five groups based on bacterial load defined by genome copies $/ \mathrm{ml} \mathrm{of} \geq 1 \times 10^{4}$; No colonisation (NC) (45.9\%), Haemophilus influenzae (HI) (17.2\%), Streptococcus pneumoniae (SP) (15.6\%), > 1 potentially pathogenic microorganism (PPM) (17.2\%) or Moraxella catarrhalis (MC) (4.1\%) ( $n=122)$. Within groups, PPM classification at 6 months follow up is shown. 53.5, 42.9, 26.3, 42.9 and 0\% of patients were recorded in the same group as baseline for no colonisation, $\mathrm{HI}, \mathrm{SP},>$ IPPM and MC respectively

associated with a lower $\mathrm{FEV}_{1}$ predicted, and a numerically higher exacerbation rate (although this difference was not statistically significant, possibly due to the small sample size, $n=9$ ). The clinical phenotype relating to persistent colonisation with $H$. influenzae is likely to be of important clinical relevance and warrants further investigation in larger longitudinal cohort analysis.

Previous studies have shown associations between total airway bacterial load and neutrophilic inflammation using sputum [6, 19] and broncho-alveolar lavage [20] samples. $H$. influenzae presence in stable state sputum samples has been associated with increased sputum neutrophil counts [21, 22] and other pro-inflammatory biomarkers [19]. The present study confirms these observations regarding $H$. influenzae, and extends our knowledge by showing that $H$. influenzae persists in the stable state in a COPD subgroup, and is associated with persistent sputum neutrophilic inflammation and a skewing away from eosinophilic inflammation. Furthermore, we show an association between $H$. influenzae and systemic neutrophilic inflammation. Increased systemic inflammation is associated with co-morbidities including cachexia 


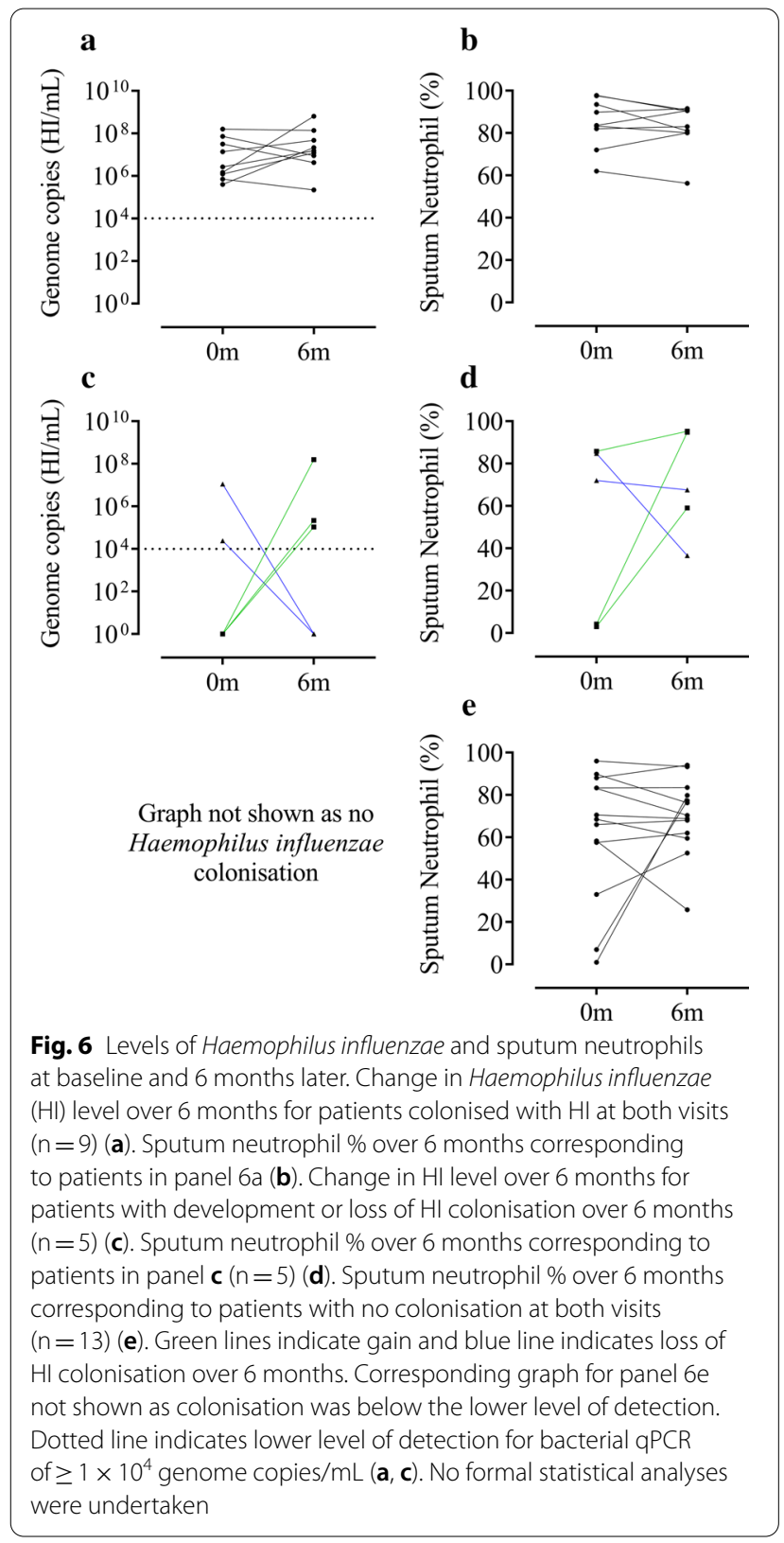

and cardiovascular disease, highlighting the potential clinical importance of our findings [23, 24].

Some COPD studies have shown that greater neutrophilic airway inflammation in the stable state is associated with poor clinical outcomes [25], including $\mathrm{FEV}_{1}$ decline [26], while other studies have reported little convincing evidence of any relationship [27]. Perhaps the variation between studies is due to differences between populations in the extent of bacterial colonisation with $H$. influenzae which is known to increase exacerbation susceptibility, particularly when exposure to human rhinovirus occurs [28].
Clinical trials have shown that lower sputum eosinophil numbers are associated with a reduced response to corticosteroid treatment [29]. The clinical use of blood eosinophils to direct ICS treatment is based on the association between blood and lung eosinophil numbers [12, 13]. Our findings implicate $H$. influenzae presence as a determinant of ICS response, as this bacterium skews the airway inflammation profile away from eosinophilic inflammation which is more ICS responsive. Furthermore, it has recently been reported that lower blood eosinophil counts are associated with increased chronic bacterial infection [30]. H. influenzae causes secretion of CXCL8 (a neutrophil chemokine) from alveolar macrophages in a corticosteroid insensitive manner in response to exposure [31], potentially explaining the observed skewing of neutrophil relative to eosinophil numbers.

$H$. influenzae can persist in the lung through various evasive pathogenic features, for example by formation of biofilms and resistance to neutrophil extracellular traps [32, 33]. The strains of $H$. influenzae present in the lungs of COPD patients may change over time [34]; our analysis does not define whether $H$. influenzae persistence at both baseline and 6 months are the same or different strains. Regardless, increases in neutrophilic airway inflammation associated with $H$. influenzae colonisation at baseline and 6 month visits were similar.

It has been reported that increased blood neutrophil numbers are associated with increased pneumonia risk in COPD patients [35]. These reports are compatible with the links between infection, microbiome dysbiosis and systemic inflammation reported here. Although we report only a relative increase in systemic neutrophil levels, the neutrophil-lymphocyte ratio (NLR) has received growing interest as a marker of systemic inflammation [36], and as a marker of severity for sepsis, pneumonia and cardiovascular diseases [36, 37]. We found the NLR to be significantly higher in those colonised with $\mathrm{H}$. influenzae, further supporting a role for the species in driving systemic inflammation and pneumonia risk.

$M$. catarrhalis has been previously described as an exacerbation-related opportunistic pathogen $[5,10]$. We report a lower stable state prevalence of $M$. catarrhalis (8.1\%) than previous studies reporting $16 \%$ and $11 \%$ prevalence $[38,39]$. These previous studies reported greater exacerbation frequencies prior to sampling (mean/year 3.6 and 3.5 [38, 39] compared with 1.89 in our analysis), possibly explaining the differences.

A limitation of this study is the reduced sample size during repeat sampling due to patient drop outs, exacerbations causing delayed stable visits and failure to produce a sputum sample at every visit. A large proportion of sputum samples analysed were spontaneously 
produced (83\% and $88 \%$ at baseline and 6 months respectively). Previous studies have reported no differences in sputum cell percentages between induced and spontaneous samples $[6,17,40]$, with our results agreeing with these findings. We observed a higher total cell count, and total neutrophil and macrophage counts in induced samples. Some caution should be applied not to over-interpret these findings, as the number of induced samples was much smaller, and no difference in total cell counts has been previously reported when paired samples from the same subjects were analysed [40]. Furthermore, this finding for total cell counts was not associated with any differences in bacteriology data. Nevertheless, our findings suggest the possibility that sputum induction results in more cellular samples compared to spontaneously obtained sputum, and this may have been a source of variation in this study [41]. Also, a large proportion of patients reported current use of ICS. It has been reported that higher ICS doses are significantly associated with greater total airway bacterial load [4, 7], but we did not see a difference in ICS use between groups, perhaps due to the high overall ICS use. Some patients were current smokers (34\%); a previous analysis of the COPDMAP data reports no difference in microbiome between smoking and non-smoking individuals [42]. We were unable to determine if any changes in colonisation status were affected by antibiotics or prior vaccination. Finally, our analyses did not extend to investigation of the molecular mechanisms pertaining to the relationship between $H$. influenzae colonisation and neutrophilic inflammation.

\section{Conclusion}

In conclusion, we observed a subgroup of COPD patients with persistent $H$. influenzae colonisation; this was associated with increased airway and systemic neutrophilic airway inflammation, and less eosinophilic airway inflammation. Although these results do not confirm causality, they highlight the possibility that $H$. influenzae may have a unique influence on inflammatory cells of the airway and circulation in COPD patients.

\section{Supplementary information}

Supplementary information accompanies this paper at https://doi. org/10.1186/s12931-020-01552-4.

Additional file 1: Figure S1. Flow chart of subject selection process. Figure S2. The comparison of Blood Lymphocyte counts between patients colonised with different PPMs at 6 months post-baseline. Figure S3. The comparison of sputum and blood eosinophil and neutrophil counts between patients colonised with different PPMs. Figure S4. The comparison of sputum and blood eosinophil and neutrophil counts between patients colonised with different PPMs at 6 months post-baseline. Figure S5. Sputum microbiome profiles in COPD patients at 6 months. Figure S6. Stability of Haemophilus presence over 6 months as defined by $16 \mathrm{~s}$ rRNA Sequencing. Table S1. Composition of > 1PPM group at baseline.
Table S2. Sputum inflammatory cell counts and qPCR for patients with frequent or infrequent exacerbations in the 12 months prior to baseline. Table S3. Baseline sputum inflammatory cell counts for induced versus spontaneous sputum samples. Table S4. Baseline sputum inflammatory cell counts for different PPM groups. Table S5. Baseline Demographics of patients enrolled onto this study for different PPM groups. Table S6. Baseline blood inflammatory cell counts for different PPM groups. Table S7. Sputum inflammatory cell counts for different PPM groups at 6 months. Table S8. Blood inflammatory cell counts for different PPM groups at 6 months. Table S9. Baseline sputum inflammatory cell counts for different PPM groups, $\times 10^{6}$ copies $/ \mathrm{ml}$ to detect PPMs. Table S10. Baseline blood inflammatory cell counts for different PPM groups, $\times 10^{6}$ copies $/ \mathrm{ml}$ to detect PPMs. Table S11. Sputum inflammatory cell counts for different PPM groups at 6 months, $\times 10^{6}$ copies $/ \mathrm{ml}$ to detect PPMs. Table S12. Blood inflammatory cell counts for different PPM groups at 6 months, $\times 10^{6} \mathrm{copies} / \mathrm{ml}$ to detect PPMs. Table S13. Baseline demographics, sputum and blood inflammatory cell counts for different PPM groups, $\times 10^{6}$ copies/ml to detect PPMs. Table S14. Baseline demographics for different PPM groups. Table S15. Sputum and blood inflammatory cell counts for different PPM groups. Table S16. Baseline demographics for different PPM groups, including those with $\mathrm{H}$. influenzae $+\geq 1$ PPM. Table S17. Baseline sputum inflammatory cell counts for different PPM groups, including those with H.influenzae $+\geq 1$ PPM. Table S18. Occurrence and average relative abundance of contaminate genera detected in sequenced negative 'blank' controls by Salter et al. [5] in the COPDMAP dataset.

\section{Abbreviations}

COPD: Chronic obstructive pulmonary disease; CAT: COPD assessment test; DCC: Differential cell count; FEV : Forced expiratory volume in $1 \mathrm{~s}$; GOLD: Global initiative for the management of chronic obstructive pulmonary disease; H. influenzae: Haemophilus influenzae; ICS: Inhaled corticosteroids; M. catarrhalis: Moraxella catarrhalis; NLR: Neutrophil-lymphocyte ratio; NC: No colonisation; PPM: Potentially pathogenic microorganism; qPCR: Quantitative polymerase chain reaction; S. pneumoniae: Streptococcus pneumoniae; SGRQ: St George's respiratory questionnaire.

\section{Acknowledgements}

Dave Singh, Augusta Beech and Simon Lea are supported by the National Institute for Health Research (NIHR) Manchester Biomedical Research Centre (BRC). We thank Dr. James R Brown, GlaxoSmithKline, for his advice in writing the manuscript.

\section{Authors' contributions}

$A B$ and $D S$ were responsible for the concept and design of study. $A B, U K, Z W$, $G D, J W, C B$, DS were involved in data acquisition. $A B$ analysed the data and DS oversaw all analyses. AB, SL, ZW and DS were responsible for data interpretation and drafting the manuscript. UK, ZW, GD, JW, CB and BM revised the manuscript critically for intellectual content. All authors have approved the final version to be published and are jointly accountable for all aspects of the work. All authors read and approved the final manuscript.

\section{Funding}

This work was supported by the Medical Research Council (MRC); COPD MAP consortium; National Institute for Health Research Respiratory and Allergy Clinical Research Facility at Manchester University NHS Foundation Trust; National Institute for Health Research (NIHR) Leicester Respiratory Biomedical Research Unit and National Institute for Health Research (NIHR) Respiratory Biomedical Research Unit at the Royal Brompton and Harefield NHS Foundation Trust and Imperial College, London UK. The views expressed in this publication are those of the author(s) and not necessarily those of the NHS, the National Institute for Health Research or the Department of Health.

Availability of data and materials

The datasets generated and/or analysed during the current study are not publicly available. 


\section{Ethical approval and consent to participate}

All patients provided written informed consent using protocols approved by the local Ethics Committees at each site (Hampstead, 11/L0/1630; Greater Manchester South, 10/H/1003/108; East Midlands, 07/H0406/157).

\section{Consent for publication}

Not applicable.

\section{Competing interests}

$A B, S L, U K$ and ZW have no competing interests. BEM is a currently employee and shareholder of GSK. GD has received personal fees from Astra-Zeneca, American Thoracic Society, FWO, Belgium. JAW has received meeting expenses and grants from GSK, Johnson and Johnson, Novartis, Boehringer Ingelheim and Astra Zeneca. CEB has received grants or consultancy paid via his institution from MRC, GSK, AZ, Novartis, Pfizer AZ/Medlmmune, Novartis, Chiesi, BI, 4DPharma, Mologic, Roche/Genentech, Sanofi and Regeneron. DS has received sponsorship to attend international meetings, honoraria for lecturing or attending advisory boards and research grants from various pharmaceutical companies including AstraZeneca, Boehringer Ingelheim, Chiesi, Cipla, Genentech, GlaxoSmithKline, from Glenmark, Gossamerbio, Menarini, Mundipharma, Novartis, Peptinnovate, Pfizer, Pulmatrix, Theravance and Verona.

\section{Author details}

${ }^{1}$ Division of Infection, Immunity and Respiratory Medicine, School of Biological Sciences, Faculty of Biology, Medicine and Health, Manchester Academic Health Science Centre, The University of Manchester, Manchester, UK.

${ }^{2}$ Medicines Evaluation Unit, University of Manchester, Manchester University NHS Foundation Trust, Southmoor Road, Manchester M23 9QZ, UK. ${ }^{3}$ Institute of Ecological Sciences, School of Life Sciences, South China Normal University, Guangzhou, China. ${ }^{4}$ Medical Innovation, Value Evidence and Outcomes, GSK R\&D, Collegeville, PA, USA. ${ }^{5}$ National Heart and Lung Institute, Imperial College London, London, UK. ${ }^{6}$ Institute for Lung Health, University of Leicester, Leicester, UK.

\section{Received: 2 July 2020 Accepted: 20 October 2020}

Published online: 01 November 2020

\section{References}

1. Singh D, Agusti A, Anzueto A, Barnes PJ, Bourbeau J, Celli BR, et al. Global strategy for the diagnosis, management, and prevention of chronic obstructive lung disease: the GOLD science committee report 2019. Eur Respir J. 2019;53(5):1900164

2. Taylor AE, Finney-Hayward TK, Quint JK, Thomas CM, Tudhope SJ, Wedzicha JA, et al. Defective macrophage phagocytosis of bacteria in COPD. Eur Respir J. 2010;35(5):1039-47.

3. Wang Z, Singh R, Miller BE, Tal-Singer R, Van Horn S, Tomsho L, et al. Sputum microbiome temporal variability and dysbiosis in chronic obstructive pulmonary disease exacerbations: an analysis of the COPDMAP study. Thorax. 2018;73(4):331-8.

4. Garcha DS, Thurston SJ, Patel AR, Mackay AJ, Goldring JJ, Donaldson GC, et al. Changes in prevalence and load of airway bacteria using quantitative PCR in stable and exacerbated COPD. Thorax. 2012;67(12):1075-80.

5. Wilkinson TMA, Aris E, Bourne S, Clarke SC, Peeters M, Pascal TG, et al. A prospective, observational cohort study of the seasonal dynamics of airway pathogens in the aetiology of exacerbations in COPD. Thorax. 2017;72(10):919-27.

6. Kolsum U, Donaldson GC, Singh R, Barker BL, Gupta V, George L, et al. Blood and sputum eosinophils in COPD; relationship with bacterial load. Respir Res. 2017;18(1):88.

7. Contoli M, Pauletti A, Rossi MR, Spanevello A, Casolari P, Marcellini A, et al. Long-term effects of inhaled corticosteroids on sputum bacterial and viral loads in COPD. Eur Respir J. 2017;50(4):1700451.

8. Ghebre MA, Pang PH, Diver S, Desai D, Bafadhel M, Haldar K, et al. Biological exacerbation clusters demonstrate asthma and chronic obstructive pulmonary disease overlap with distinct mediator and microbiome profiles. J Allergy Clin Immunol. 2018;141(6):2027-36 e12.
9. Wang Z, Bafadhel M, Haldar K, Spivak A, Mayhew D, Miller BE, et al. Lung microbiome dynamics in COPD exacerbations. Eur Respir J. 2016:47(4):1082-92.

10. Wang Z, Maschera B, Lea S, Kolsum U, Michalovich D, Van Horn S, et al. Airway host-microbiome interactions in chronic obstructive pulmonary disease. Respir Res. 2019;20(1):113.

11. Singh R, Mackay AJ, Patel AR, Garcha DS, Kowlessar BS, Brill SE, et al. Inflammatory thresholds and the species-specific effects of colonising bacteria in stable chronic obstructive pulmonary disease. Respir Res. 2014; $15: 114$

12. Singh D, Bafadhel M, Brightling CE, Sciurba FC, Curtis JL, Martinez FJ, et al. Blood eosinophil counts in clinical trials for chronic obstructive pulmonary disease. Am J Respir Crit Care Med. 2020;202(5):660-71.

13. Kolsum U, Damera G, Pham TH, Southworth T, Mason S, Karur P, et al. Pulmonary inflammation in patients with chronic obstructive pulmonary disease with higher blood eosinophil counts. J Allergy Clin Immunol. 2017;140(4):1181-4.

14. Jones PW, Harding G, Berry P, Wiklund I, Chen WH, Kline LN. Development and first validation of the COPD Assessment Test. Eur Respir J. 2009;34(3):648-54.

15. Jones PW, Quirk FH, Baveystock CM, Littlejohns P. A self-complete measure of health status for chronic airflow limitation. The St. George's Respiratory Questionnaire. Am Rev Respir Dis. 1992;145(6):1321-7.

16. Miller MR, Hankinson J, Brusasco V, Burgos F, Casaburi R, Coates A, et al. Standardisation of spirometry. Eur Respir J. 2005;26(2):319-38.

17. Pizzichini MM, Popov TA, Efthimiadis A, Hussack P, Evans S, Pizzichini E, et al. Spontaneous and induced sputum to measure indices of airway inflammation in asthma. Am J Respir Crit Care Med. 1996;154(4 Pt 1):866-9.

18. Bafadhel M, McCormick M, Saha S, McKenna S, Shelley M, Hargadon $B$, et al. Profiling of sputum inflammatory mediators in asthma and chronic obstructive pulmonary disease. Respiration. 2012;83(1):36-44.

19. Tufvesson E, Bjermer L, Ekberg M. Patients with chronic obstructive pulmonary disease and chronically colonized with Haemophilus influenzae during stable disease phase have increased airway inflammation. Int J Chronic Obstruct Pulm Dis. 2015;10:881-9.

20. Sethi S, Maloney J, Grove L, Wrona C, Berenson CS. Airway inflammation and bronchial bacterial colonization in chronic obstructive pulmonary disease. Am J Respir Crit Care Med. 2006;173(9):991-8.

21. Bafadhel M, Haldar K, Barker B, Patel H, Mistry V, Barer MR, et al. Airway bacteria measured by quantitative polymerase chain reaction and culture in patients with stable COPD: relationship with neutrophilic airway inflammation, exacerbation frequency, and lung function. Int J Chronic Obstruct Pulm Dis. 2015a;10:1075-83.

22. Marin A, Monso E, Garcia-Nunez M, Sauleda J, Noguera A, Pons J, et al. Variability and effects of bronchial colonisation in patients with moderate COPD. Eur Respir J. 2010;35(2):295-302.

23. Miller J, Edwards LD, Agusti A, Bakke P, Calverley PM, Celli B, et al. Comorbidity, systemic inflammation and outcomes in the ECLIPSE cohort. Respir Med. 2013;107(9):1376-84.

24. Dima E, Kyriakoudi A, Kaponi M, Vasileiadis I, Stamou P, Koutsoukou A, et al. The lung microbiome dynamics between stability and exacerbation in chronic obstructive pulmonary disease (COPD): current perspectives. Respir Med. 2019;157:1-6.

25. Gernez $Y$, Tirouvanziam R, Chanez P. Neutrophils in chronic inflammatory airway diseases: can we target them and how? Eur Respir J. 2010;35(3):467-9.

26. Stanescu D, Sanna A, Veriter C, Kostianev S, Calcagni PG, Fabbri LM, et al. Airways obstruction, chronic expectoration, and rapid decline of FEV1 in smokers are associated with increased levels of sputum neutrophils. Thorax. 1996;51(3):267-71.

27. Singh D, Edwards L, Tal-Singer R, Rennard S. Sputum neutrophils as a biomarker in COPD: findings from the ECLIPSE study. Respir Res. 2010;11:77.

28. Hicks A, Healy E, Sandeman N, Feelisch M, Wilkinson T. A time for everything and everything in its time-exploring the mechanisms underlying seasonality of COPD exacerbations. Int J Chronic Obstruct Pulm Dis. 2018;13:2739-49.

29. Bafadhel M, Peterson S, De Blas MA, Calverley PM, Rennard SI, Richter K, et al. Predictors of exacerbation risk and response to budesonide in patients with chronic obstructive pulmonary disease: 
a post-hoc analysis of three randomised trials. Lancet Respir Med. 2018;6(2):117-26.

30. Martinez-Garcia MA, Faner R, Oscullo G, la Rosa-Carrillo D, SolerCataluna JJ, Ballester M, et al. Inhaled steroids, circulating eosinophils, chronic airway infection and pneumonia risk in chronic obstructive pulmonary disease: a network analysis. Am J Respir Crit Care Med. 2020;201(9):1078-85.

31. Khalaf RM, Lea SR, Metcalfe HJ, Singh D. Mechanisms of corticosteroid insensitivity in COPD alveolar macrophages exposed to NTHi. Respir Res. 2017;18(1):61

32. Ahearn CP, Gallo MC, Murphy TF. Insights on persistent airway infection by non-typeable Haemophilus influenzae in chronic obstructive pulmonary disease. Pathog Dis. 2017;75(4):ftx042.

33. King P. Haemophilus influenzae and the lung (Haemophilus and the lung). Clin TransI Med. 2012;1 (1):10.

34. Maddi S, Kolsum U, Jackson S, Barraclough R, Maschera B, Simpson KD, et al. Ampicillin resistance in Haemophilus influenzae from COPD patients in the UK. Int J Chronic Obstruct Pulm Dis. 2017;12:1507-18.

35. Pascoe SJ, Papi A, Midwinter D, Lettis S, Barnes N. Circulating neutrophils levels are a predictor of pneumonia risk in chronic obstructive pulmonary disease. Respir Res. 2019;20(1):195.

36. Karakioulaki M, Stolz D. Biomarkers in pneumonia-beyond procalcitonin. Int J Mol Sci. 2019;20(8):2004.

37. Liu X, Shen Y, Wang H, Ge Q, Fei A, Pan S. Prognostic significance of neutrophil-to-lymphocyte ratio in patients with sepsis: a prospective observational study. Mediat Inflamm. 2016;2016:8191254.
38. Bafadhel M, Haldar K, Barker B, Patel H, Mistry V, Barer MR, et al. Airway bacteria measured by quantitative polymerase chain reaction and culture in patients with stable COPD: relationship with neutrophilic airway inflammation, exacerbation frequency, and lung function. Int J Chronic Obstruct Pulm Dis. 2015b;10:1075-83.

39. Barker BL, Haldar K, Patel H, Pavord ID, Barer MR, Brightling CE, et al. Association between pathogens detected using quantitative polymerase chain reaction with airway inflammation in COPD at stable state and exacerbations. Chest. 2015:147(1):46-55.

40. Khurana S, Ravi A, Sutula J, Milone R, Williamson R, Plumb J, et al. Clinical characteristics and airway inflammation profile of COPD persistent sputum producers. Respir Med. 2014;108(12):1761-70.

41. Tangedal S, Aanerud M, Gronseth R, Drengenes C, Wiker HG, Bakke PS, et al. Comparing microbiota profiles in induced and spontaneous sputum samples in COPD patients. Respir Res. 2017;18(1):164.

42. Haldar K, George L, Wang Z, Mistry V, Ramsheh MY, Free RC, et al. The sputum microbiome is distinct between COPD and health, independent of smoking history. Respir Res. 2020;21(1):183.

\section{Publisher's Note}

Springer Nature remains neutral with regard to jurisdictional claims in published maps and institutional affiliations.
Ready to submit your research? Choose BMC and benefit from:

- fast, convenient online submission

- thorough peer review by experienced researchers in your field

- rapid publication on acceptance

- support for research data, including large and complex data types

- gold Open Access which fosters wider collaboration and increased citations

- maximum visibility for your research: over $100 \mathrm{M}$ website views per year

At BMC, research is always in progress.

Learn more biomedcentral.com/submissions 